\title{
Transmutation of Matter
}

[Shortly before his death on October 19 last year, Lord Rutherford completed the presidential address which he proposed to deliver at the meeting of the Indian Science Congress Association on January 3-9. The latter part of the address is here reproduced and represents Lord Rutherford's last pronouncement on a subject with which his name will always be associated. Other matters discussed by him in the address were referred to in last week's leading article.-Editor, NATURE.]

$\mathrm{T}$ OWARDS the close of the nineteenth century, when it seemed certain that the atoms of the elements were unchangeable by the forces then at our command, a discovery was made which has revolutionized our conception of the nature and relations of the elements. I refer to the discovery in 1896 of the radioactivity of the two heaviest elements, uranium and thorium. It was soon made clear that this radioactivity is a sign that the atoms of these elements are undergoing spontaneous transmutation. At any moment, a small fraction of the atoms concerned become unstable and break up with explosive violence, hurling out either a charged atom of helium, known as an $\alpha$-particle, or a swift electron of light mass called a $\beta$-particle. As a result of these explosions, a new radioactive element is formed, and the process of transmutation once started continues through a number of stages. Each of the radioactive elements formed in this way breaks up according to a simple universal law but at very different rates. In a surprisingly short time, these successive transformations were disentangled and more than thirty new types of elements brought to light, while the simple chemical relations between them were soon made clear.

We had thus been given a vision of a new and startling sub-atomic world where atoms break up spontaneously with an enormous release of energy quite uninfluenced by the most powerful agencies at our disposal. Apart from uranium and thorium and the elements derived from them, only a few other elements showed even a feeble trace of radioactivity. The great majority of our ordinary elements appeared to be permanently stable under ordinary conditions on our earth. Science was then faced with the problem whether artificial methods could be found to transmute the atoms of the ordinary elements. Before this problem could be attacked with any hope of success, it was necessary to know more of the actual constitution of atoms. This information was provided by the rise of the nuclear theory of atomic structure, which I first suggested in 1911. The essential controlling feature of all atoms was found to reside in a very minute central nucleus which carried a positive charge and contained most of the mass of the atom. A relation of unexpected simplicity was found to connect the atoms of all the elements. The ordinary properties of an atom are defined by a whole number, which represents the number of units of resultant positive charge carried by the nucleus. This varies from 1 for hydrogen to 92 for the heaviest element uranium, and, with few exceptions, all the intervening numbers correspond to known elements.

On this view of atomic structure it was evident that, to bring about the transmutation of an atom, it was necessary in some way to alter the charge or mass of the nucleus or both together. Since the nucleus of an atom must be held together by very powerful forces of some kind, this could only be effected by bringing a concentrated source of energy of some kind to bear on the individual nucleus. The most energetic projectile available at that time was the swift $\alpha$-particle spontaneously ejected from radioactive substances. If a large number of $\alpha$-particles were fired at random at a sheet of matter, it was to be expected that one of them must occasionally approach very closely to the nucleus of any light atom in its path. In such a close encounter, the nucleus must be violently disturbed, and possibly under favourable conditions the $\alpha$-particle might actually enter the nuclear structure.

This mode of attack upon the nucleus at once proved successful. I found in 1919 that nitrogen could be transformed by bombardment with fast $\alpha$-particles. The process of transmutation is now clear. Occasionally an $\alpha$-particle actually enters the nitrogen nucleus and forms with it a new unstable nucleus which instantly breaks up with the emission of a fast proton (hydrogen nucleus) and the formation of a stable isotope of oxygen of mass 17. About a dozen of the light elements were found to be transformed in a similar way. The protons liberated in the nuclear explosions were at first counted by observing the flashes of light (scintillations) produced in phosphorescent zinc sulphide. This method was slow and very trying to the eyes of the observers. Progress, however, becæme more rapid and definite when electrical methods of counting individual fast particles were developed. These electrical counters, mainly depending on the use of electron tubes for magnifying small currents, have now reached such a stage of perfection that we are able to count automatically individual fast particles like $\alpha$ particles and protons even though they enter the 
detecting chamber at a rate as fast as ten thousand per minute. By other special devices, we are in like manner able to count individual $\beta$-particles. In this connexion, I must not omit to mention that wonderful instrument, the Wilson expansion chamber, which makes visible to us the actual tracks of flying fragments of atoms resulting from an atomic explosion. These remarkable devices have played an indispensable part in the rapid growth of knowledge during the last few years. It is to be emphasized that progress in scientific discovery is greatly influenced by the development of new technical methods and of new devices for measurement. With the growing complexity of seience, the development of special techniques is of ever-increasing importance for the advance of knowledge.

Up to the year 1932, experiments on transmutation were confined to the use of $\alpha$-particles for bombarding purposes. It became clear that the process of transformation was in most cases complex, since groups of protons with different but characteristic energies were observed when a single element was bombarded. This led to the conception that discrete energy levels existed within a nucleus, and that under some conditions part of the excess energy was sometimes released in the form of a quantum of high-frequency radiation.

The stage was now set for a great advance, and four new discoveries of outstanding importance were made in rapid succession in the period 193133. I refer to the discovery of the positive electron by Anderson in 1931, of the neutron by Chadwick in 1932, of artificial radioactivity by $M$. and Mme. Curie-Joliot in 1933 and of the transmutation of the elements by purely artificial methods first shown by Cockeroft and Walton in 1932.

The discovery of the neutron-that uncharged particle of mass nearly 1 -was the result of a close study of the effects produced in the light element beryllium when bombarded by $\alpha$-particles. It is noteworthy that the proton and neutron, which are now believed to be the essential units with which all atomic nuclei are built up, owe their recognition to a study of the transmutation of matter by $\alpha$-particles.

Before the discovery of the neutron, it had been perforce assumed that nuclei must in some way be built up of massive protons and light negative electrons. Theories of nuclear structure became much more amenable to calculation when the nucleus was considered to be an aggregate of parts like the proton and neutron which have nearly the same mass. There was no longer any need to assume that either the positive or the negative electron has an independent existence in the nuclear structure. We are still uncertain of the exact relation, if any, between the neutron and the proton. The neutron appears to be slightly more massive than the proton, but it is generally believed, although no definite proof is available, that the proton and neutron within a nucleus are mutually convertible under certain conditions. For example, the change of a proton into a neutron within the nucleus should lead to the appearance of a free positive electron, while conversely the change of a neutron into a proton gives rise to a free negative electron. In this way it appears possible to account for the observed fact that either positive or negative electrons are emitted by a large group of radioactive elements to which I will now refer.

In the early experiments on transmutation by $\alpha$-particles, it was supposed that a stable nucleus was always formed after the emission of a fast proton. The investigations of $\mathbf{M}$. and Mme. CurieJoliot showed that in some cases elements were formed which, while momentarily stable, ultimately broke up slowly, exactly like the natural radioactive bodies. Most of these radioactive bodies formed by artificial methods break up with the expulsion of fast negative electrons, but in a few cases positive electrons are emitted. Since the presence of these radioactive bodies can be easily detected, and their chemical properties readily determined, this new method of attack on the problem of transmutation has proved of great value. Nearly a hundred of these radioactive bodies are now known, produced in a great variety of ways. Some arise from the bombardment by fast $\alpha$-particles, others by bombardment with protons or deuterons. As Fermi and his colleagues have shown, neutrons, and particularly slow neutrons, are extraordinarily effective in the formation of such radioactive bodies. On account of its absence of charge, the neutron enters freely into the nuclear structure of even the heaviest element, and in many cases causes its transmutation. For example, a number of these radioactive bodies are produced when the two heaviest elements, uranium and thorium, are bombarded by slow neutrons. In the case of uranium, as Hahn and Meitner have shown, the radioactive bodies so formed break up in a succession of stages like the natural radioactive bodies, and give rise to a number of trans-uranic elements of higher atomic number than uranium (92). These radioactive elements have the chemical properties to be expected from the higher homologues of rhenium, osmium and iridium of atomic numbers 93,94 and 95.

These artificial radioactive bodies in general represent unstable varieties of the isotopes of known elements which have a limited life. No doubt such transient radioactive elements are still produced by transmutation in the furnace of our 
sun, where the thermal motions of the atoms must be very great. These radioactive elements would rapidly disappear as soon as the earth cooled down after separation from the sun. On this view, uranium and thorium are to be regarded as practically the sole survivors in our earth of a large group of radioactive elements, owing to the fact that their time of transformation is long compared with the age of our planet.

It is of interest to note what an important part the $\alpha$-particle, which is itself a product of transformation of the natural radioactive bodies, has played in the growth of our knowledge of artificial transmutation. It is to be remembered, too, that our main source of neutrons for experimental purposes is provided by the bombardment of beryllium with $\alpha$-particles. The amount of radium available in our laboratories is, however, limited, and it was early recognized that if our knowledge of transmutation was to be extended, it was necessary to have a copious supply of fast particles of all kinds for bombarding purposes. It is well known that enormous numbers of protons and deuterons, for example, can be easily produced by the passage of the electric discharge through hydrogen and deuterium (heavy hydrogen). To be effective for transmutation purposes, however, these charged particles must be given a high speed by accelerating them in a strong electric field. This has involved the use of apparatus on an engineering scale to provide voltages as high as one million volts or more, and the use of fast pumps to maintain a good vacuum.

A large amount of difficult technical work has been necessary to produce such high D.c. voltages and to find the best methods of applying them to the accelerating system. In Cambridge, these high voltages are produced by multiplying the voltage of a transformer by a system of condensers and rectifiers ; in the United States of America by the use of a novel type of electrostatic generator, first developed by van der Graaf. Prof. Lawrence, of the University of California, has devised an ingenious instrument called a 'cyclotron' in which the charged particles are automatically accelerated in multiple stages. This involves the use of huge electromagnets and very powerful electric oscillators. By this method he has succeeded in producing streams of fast particles which have energies as high as the $\alpha$-particle ejected from radioactive substances. Undoubtedly this type of apparatus will prove of great importance in giving us a supply of much faster particles than we can hope to produce by the more direct methods.

It was at first thought that very high potentials of the order of several million volts would be required to obtain particles to study the transmutation of elements. Here, however, the develop- ment of the theory of wave-mechanics came to the aid of the experimenter, for Gamow showed that there was a small chance that comparatively slow bombarding particles might enter a nucleus. This theoretical conclusion has been completely verified by experiment. In the case of a light element like lithium, transformation effects can be readily observed with protons of energy as low as 20,000 volts. Of course, the amount of transformation increases rapidly with rise of voltage.

The study of the transmutation of elements by using accelerated protons and deuterons as bombarding particles has given us a wealth of new information. The capture of the proton or deuteron by a nucleus leads in many cases to types of transmutation of unusual interest. For example, the bombardment of the isotope of lithium of mass 7 by protons leads to the formation of a beryllium nucleus of mass 8 with a great excess of energy. This immediately breaks up into two $\alpha$-particles shot out in nearly opposite directions. When boron $\mathrm{Il}$ is bombarded by protons, a carbon nucleus of mass 12 is formed which breaks up in most cases into three $\alpha$-particles. The deuteron is in some respects even more effective than the proton as a transmuting agent. When deuterons are used to bombard a compound of deuterium, previously unknown isotopes of hydrogen and of helium of mass 3 are formed, while fast protons and neutrons are liberated. The bombardment of beryllium by very fast deuterons gives rise to a plentiful supply of neutrons. Lawrence has shown that the bombardment of bismuth by very fast deuterons leads to the production of a radioactive bismuth isotope which is identical with the wellknown natural radioactive product radium $\mathrm{E}$. Many artificial radioactive elements can be produced, often in great intensity. For example, the bombardment of common salt by fast deuterons gives rise to a radioactive isotope of sodium. This breaks up with a half period of 15 hours, emitting not only fast $\beta$-particles but also $\gamma$-rays at least as penetrating as those from radium.

It may well be that in course of time such artificial radioactive elements may prove a useful substitute for radium in therapeutic work. By these methods also, such intense sources of neutrons can be produced that special precautions have to be taken for the safety of the operators of the apparatus.

Sufficient I think has been said to illustrate the variety and interest of the transmutations produced by these bombardment methods. It should, however, be pointed out that transmutation in some cases can be effected by transferring energy to a nucleus by means of $\gamma$-rays of high quantum energy instead of by a material particle. For example, the deuteron can be broken up into its 
components, the proton and neutron, by the action of the $\gamma$-rays from radium or thorium. As a result of the bombardment of lithium by protons, $\gamma$-rays of extraordinarily great quantum energy as high as 17 million volts are strongly emitted. Bothe has recently shown that these high-energy rays are able to transmute a number of atoms, neutrons usually being emitted in the process.

Some simple laws appear to hold in all individual transformations so far examined. Nuclear charge is always conserved, and where heavy particles are emitted, so also is energy when account is taken of the equivalence of mass and energy. Certain difficulties arise with regard to the conservation of energy in cases where light positive and negative electrons are emitted during transmutation, and there is still much discussion on this important question.

The study of the transmutation of matter has been extraordinarily fruitful in results of fundamental importance. In addition to the $\alpha$-particle, it has disclosed to us the existence of those two building units of nuclei, the proton and neutron. It has greatly widened our conception of the varieties of atomic nuclei which can exist in Nature. Not only has it led to the discovery of about one hundred new radioactive elements, but also of several stable isotopes of known elements, like ${ }^{3} \mathrm{H},{ }^{3} \mathrm{He},{ }^{8} \mathrm{Be}$, which had previously been unsuspected. It has greatly extended our knowledge of the ways in which nuclei can be built up and broken down, and has brought to our attention the extraordinary violence of some of the nuclear explosions which occur. The great majority of our elements have been transmuted by the bombardment method, and in the ease of the light elements which have been most carefully studied, a great variety of modes of transmutation has been established.

Rapid progress has been made, but much still remains to be done before we can hope to understand the detailed structure and stability of different forms of atomic nuclei and the origin of the elements. I cannot but reflect on the amazing contrast between my first experiment on the transmutation of nitrogen in the University of Manchester in 1919 and the large-scale experiments on transmutation which are now in progress in many parts of the world. In the one case, imagine an observer in a dark room with very simple apparatus painfully counting with a microscope a few faint scintillations originating from the bombardment of nitrogen by a source of $\alpha$-particles. Contrast this with the large-scale apparatus now in use for experiments on transmutation in Cambridge. A great hall contains massive and elaborate machinery, rising tier on tier, to give a steady potential of about two million volts. Nearby is the tall accelerating column with a power station on top, protected by great corona shields-reminding one of a photograph in the film of Wells's "The Shape of Things to Come". The intense stream of accelerated particles falls on the target in the room below, with thick walls to protect the workers from stray radiation. Here is a band of investigators using complicated electrical devices for counting automatically the multitude of fast particles arising from the transformation of the target element, or photographing with an expansion chamber, automatically controlled, the actual tracks of particles from exploding atoms.

To examine the effect of still faster particles, a cyclotron is installed in another large room. The large electromagnet and accessories are surrounded with great water tanks containing boron in solution to protect the workers from the effect of neutrons released in the apparatus. A power station nearby is needed to provide current to excite the electromagnet and the powerful electric oscillators.

Such a comparison illustrates the remarkable changes in the scale of research that have taken place in certain branches of pure science within the last twenty years. Such a development is inevitable, for, as science progresses, important problems arise which can only be solved by the use of large powers and complicated apparatus, requiring the attention of a team of research workers. If rapid progress is to be made, such team work is likely to be a feature of the more elaborate researches in the future. Fortunately there is still plenty of scope for the individual research worker in many experiments of a simpler kind.

The science of physics now covers such a vast field that it is impossible for any laboratory to provide up-to-date facilities for research in more than a few of its branches. There is a growing tendency in our research laboratories to-day to specialize in those particular branches of physics in which they are most interested or specially equipped. Such a division of the field of research amongst a number of universities has certain advantages, provided that this subdivision is not carried too far. In general, the universities should be left free so far as possible to develop their own lines of research and encouraged to train young investigators, for it cannot be doubted that vigorous schools of research in pure science are vital to any nation if it wishes to develop effectively the application of science, whether to agriculture, industry or medicine. Since investigations in modern science are sometimes costly, and often require the use of expensive apparatus and largescale collaboration, it is obviously essential that adequate funds should be available to the universities to cover the cost of such researches. 